\title{
Teaching practice and effect of the curriculum design and simulation courses under the support of professional optical software
}

\section{YuanFang Lin, XiaoDong Zheng, YuJia Huang}

YuanFang Lin, XiaoDong Zheng, YuJia Huang, "Teaching practice and effect of the curriculum design and simulation courses under the support of professional optical software," Proc. SPIE 10452, 14th Conference on Education and Training in Optics and Photonics: ETOP 2017, 104520P (16 August 2017); doi: 10.1117/12.2269723

SPIE Event: 14th Conference on Education and Training in Optics and Photonics, ETOP 2017, 2017, Hangzhou, China 


\title{
Teaching practice and effect of the curriculum design and simulation courses under the support of professional optical software
}

\author{
YuanFang Lin*, XiaoDong Zheng, YuJia Huang \\ College of Optical Science and Engineering, Zhejiang University, Hangzhou 310027, China
}

\begin{abstract}
Curriculum design and simulation courses are bridges to connect specialty theories, engineering practice and experimental skills. In order to help students to have the computer aided optical system design ability adapting to developments of the times, a professional optical software--Advanced System of Analysis Program (ASAP) was used in the research teaching of curriculum design and simulation courses. The ASAP tutorials conducting, exercises both complementing and supplementing the lectures, hands-on practice in class, autonomous learning and independent design after class were bridged organically, to guide students "learning while doing, learning by doing", paying more attention to the process instead of the results. Several years of teaching practice of curriculum design and simulation courses shows that, project-based learning meets society needs of training personnel with knowledge, ability and quality. Students have obtained not only skills of using professional software, but also skills of finding and proposing questions in engineering practice, the scientific method of analyzing and solving questions with specialty knowledge, in addition, autonomous learning ability, teamwork spirit and innovation consciousness, still scientific attitude of facing failure and scientific spirit of admitting deficiency in the process of independent design and exploration.
\end{abstract}

Keywords: Higher education, Optical engineering, Optics and photonics, Undergraduate program, Curriculum design, Modeling and simulation, Teaching reform, Project-based learning

\section{INTRODUCTION}

The undergraduate major of Optics and Photonics (O \& P) of Zhejiang University was founded in 1952. The major name had changed several times. It was named Optical Instrument from 1952 to 1996, Optical Information Engineering from 1997 to 2005, Information Engineering (optics and photonics direction) from 2006 to 2012, and Optical Information Science and Engineering from 2013 till now. Optics and photonics program changed with the evolution of major names. Curriculum design and simulation courses are bridges to connect specialty theories, engineering practice and experimental skills. There was a required course for the-fourth-year students called "curriculum design of optical systems" in the 2006-2012 O \& P undergraduate program, the credit of which is 1.25 . There was an elective course for the-third-year students called "Modeling and simulation of optical components and systems" in $\mathrm{O} \& \mathrm{P}$ undergraduate program since 2013, the credit of which is 1.5 . Teaching practice and effect of those curriculum design and simulation courses under the support of professional optical software are presented in this paper.

*linyuanfang@zju.edu.cn; phone 86571 8795-1681; fax 86571 8795-1681;

14th Conference on Education and Training in Optics and Photonics: ETOP 2017, edited by Xu Liu,

Xi-Cheng Zhang, Proc. of SPIE Vol. 10452, 104520P · C 2017 ICO, IEEE, OSA, SPIE

CCC code: $0277-786 \mathrm{X} / 17 / \$ 18 \cdot$ doi: $10.1117 / 12.2269723$

Proc. of SPIE Vol. 10452 104520P-1 


\section{A PROFESSIONAL OPTICAL SOFTWARE -- ASAP}

ASAP is Advanced Systems Analysis Program for short. It is developed and sold by Breault Research organization (BRO) in America, commercially available for over 30 years. ASAP is fundamentally a flexible and efficient optical system-modeling tool. It is able to simulate, by Monte Carlo ray-tracing techniques, the interaction of light with optical and mechanical structures. It does this in a single, global, three-dimensional coordinate system, making no assumptions about symmetry. Rays can automatically split into reflected, refracted, diffracted, polarized, and scattered components as they propagate through the system. The rays proceed independently, following physically realizable paths, encountering objects in any order, as appropriate. This type of ray tracing is often described using terms like "unconstrained" or "non-sequential". What ASAP is really doing, however, is just a simulation, based on the way that real light behaves in the real world. ASAP can also simulate coherent and diffractive optical systems with a relatively simple but powerful extension of ray tracing methods known as Gaussian beam decomposition. Any complex field can be decomposed into a set of Gaussian beams that in turn can be traced as described above by ray-trace methods. This allows users to work on problems involving coherence, diffraction, interference, coupling efficiency, and other situations where phase matters ${ }^{[1]}$.

\section{ASAP TEACHING PRACTICE IN ZHEJIANG UNIVERSITY}

ASAP was permitted to sell to China since 2004 and firstly introduced into the graduate classroom teaching of Zhejiang University in winter 2007. The course is named "simulation of optical systems based on ASAP", which has been opened for graduates majoring in Optical Engineering of Zhejiang University ten semesters so far. A course named "curriculum design of optical systems" was opened for undergraduates majoring in optometry of Wenzhou Medical College in spring 2009 and spring 2010. The course also had been opened for undergraduates majoring in Information Engineering (optics and photonics direction) of Zhejiang University six semesters from winter 2009 to winter 2014. In addition, a course named "modeling and simulation of optical components and systems" had been opened for undergraduates majoring in Optical Information Science and Engineering of Zhejiang University two semesters from fall 2015 to fall 2016. ASAP was used in research teaching of the above three courses and the number of students was limited to 42 each semester. The textbook adopted was ASAP Primer. The ASAP tutorials conducting, exercises complementing and supplementing the lectures, hands-on practice in class, autonomous learning and independent design after class were bridged organically, to guide students "learning while doing, learning by doing", paying more attention to the process instead of the results during the implementation of performance evaluation.

\section{STUDENTS' RESEARCH PROJECTS BASED ON ASAP}

Students of the above undergraduate courses had been required to form a team of 1,2 or 3 members, to do a research project based on ASAP during the semester and to present it in the final examination. Table 1 shows the quantity of students' research projects based on ASAP each semester so far. The total number is 156. Some projects among them are about the modeling, simulation and analysis of optical phenomena including reflection, refraction, diffraction, polarization, scattering, coherence and so on. The other projects are concerned with the radiometry, diffuser, optimization of LED and auto lamp design, etc.

Table 1. The quantity of students' research projects based on ASAP each semester so far

\begin{tabular}{|c|c|c|c|c|c|c|c|c|}
\hline Winter 2009 & Spring 2010 & Winter 2010 & Winter 2011 & Winter 2012 & Winter 2013 & Winter 2014 & Fall 2015 & Fall 2016 \\
\hline 41 & 13 & 17 & 16 & 16 & 9 & 14 & 15 & 15 \\
\hline
\end{tabular}


Based on some students' research projects and experimental equipment of a fourth-year course "optical information lab", we simulated the conoscopic interference in uniaxial crystal under different beam divergence angles (see Figure 1) ${ }^{[2]}$, implemented the simulation analysis and experimental verification of the optical image addition and subtraction (see Figure 2) ${ }^{[3]}$, and of the holographic grating (see Figure 3$)^{[4]}$.

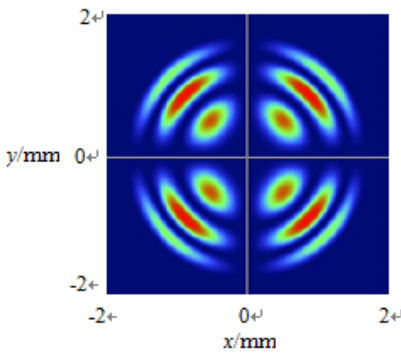

(a) $36^{\circ}$

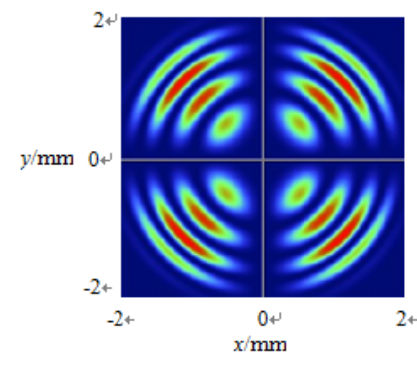

(b) $44^{\circ}$

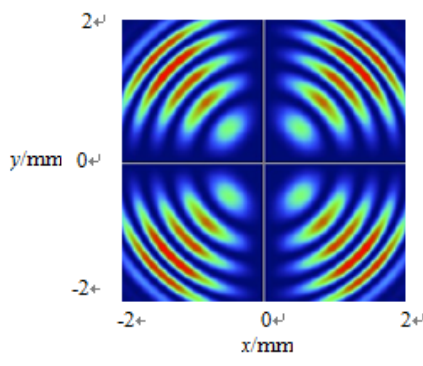

(c) $52^{\circ}$

Figure 1. Conoscopic interferograms under different divergence angles.

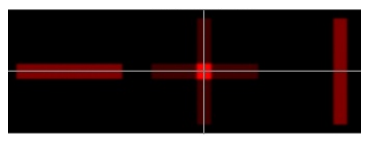

(a) Simulation phenomena

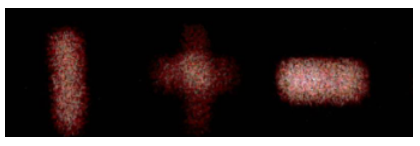

(b) Experimental phenomena

Figure 2. Simulation and experimental verification of the optical image addition and subtraction

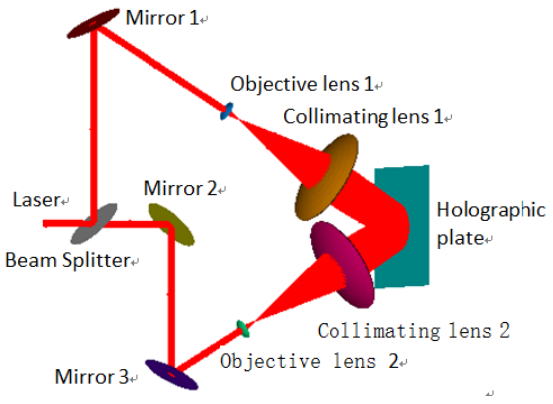

(a) System modeling

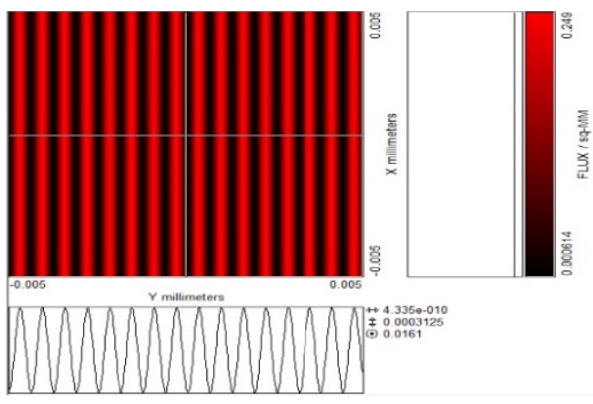

(b) Simulation interferogram

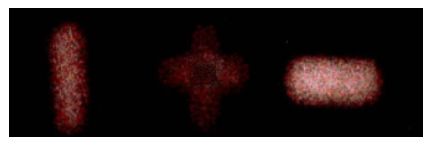

(1)

(1)

Figure 3. Simulation and experimental verification of the holographic grating

Here we present a detail example of students' research project based on ASAP. A student team chose to simulate macroscopic invisible cloak structure proposed in reference [5]. The main obstacle that prevent macroscopic invisible cloak to be realized in practical device is phase preservation requirement. But for observation with human eyes, this requirement can be abandoned due to incoherent, non-polarization sensitive and broadband properties. The team adopted the simplified version of invisible cloak, using ray optics model and isotropic materials, with the help of ASAP. To construct the cloak in ASAP, there are two key problems that need to be solved. One is the geometry parameters of cloak structure, the other is the refraction index of glasses. The team's code will calculate all the geometry parameters according to the number of edges set by users. Then the students divide the planes into different groups and automatically construct the cloak by rotating the reference plane. To determine the refraction index, one way is to list the equations and solve for a universal analytical solution for structures with arbitrary number of edges, however, it is a transcendental equation which is difficult to solve. So they decided to use binary search to optimize the refraction index. 
Figure 4 shows different output ray direction under different refraction indexes. Only when the refraction index is optimal, the output ray direction is horizontal, which can serve as the criterion in binary search algorithm.

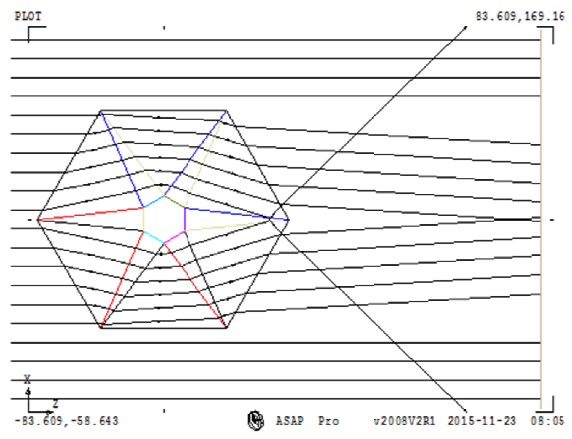

(a) RI > Optimal RI

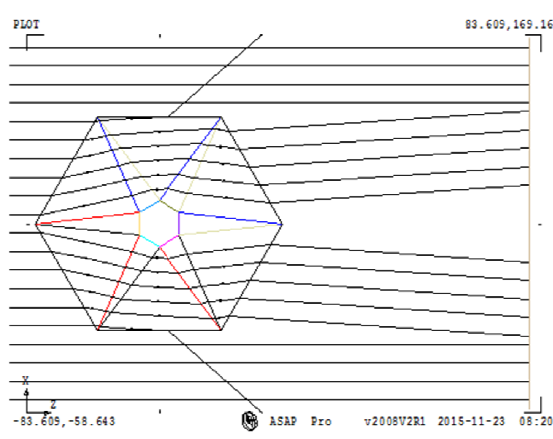

（b） RI < Optimal RI

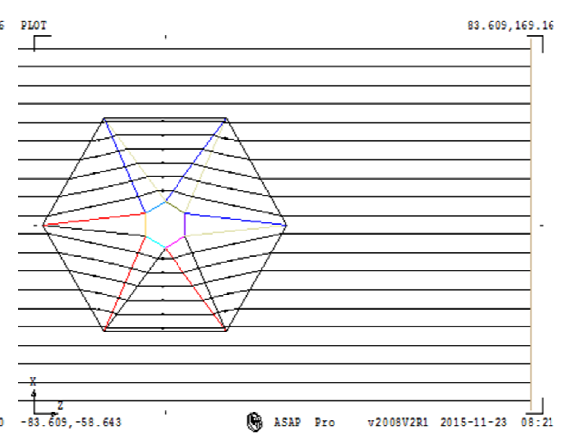

(c) Optimal RI

Figure 4. Output ray direction under different refraction indexes in a hexagon cloak

The students test the performance of macroscopic invisible cloak. The first evaluation criterion is propagation direction of output rays. As is shown in Figure 5, after refraction index optimization, the propagation direction of output rays remains horizontal for structure with various numbers of edges. It is noticed that when the number of edges increases, a smaller portion of input light remains previous propagation direction, which will lower the power of light reaching the detector. They further test the fault tolerance performance of invisible cloaks with different shapes by letting the incident angle slightly deviate from horizontal angle. They set flux efficiency to be one when incident angle deviation is $0^{\circ}$. As Figure 6 shows, the flux deviation decreases almost linearly with the deviation degrees.

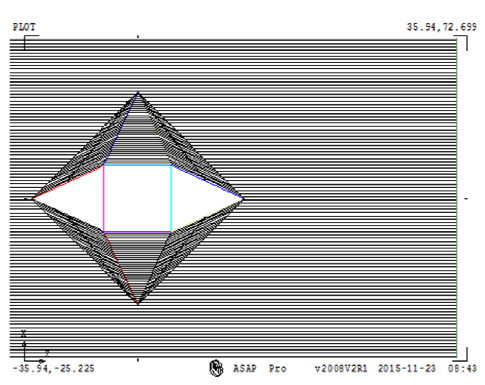

(a) Quadrangle structure, $\mathrm{n}=1.769033$

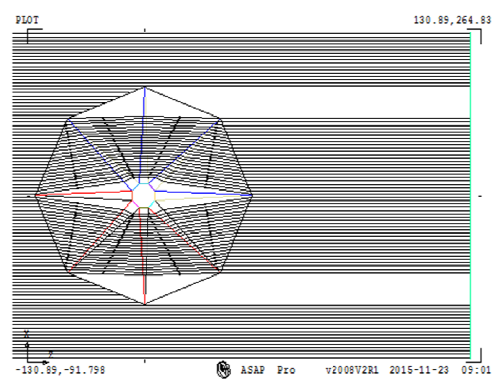

(b) Octagon structure, $\mathrm{n}=1.874375$

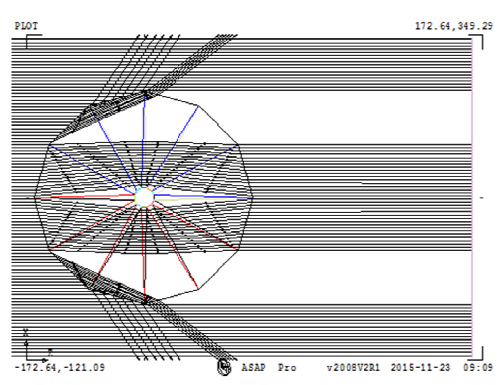

(c) Dodecagon structure, $\mathrm{n}=1.394127$

Figure 5. Output ray direction of cloaks with different number of edges

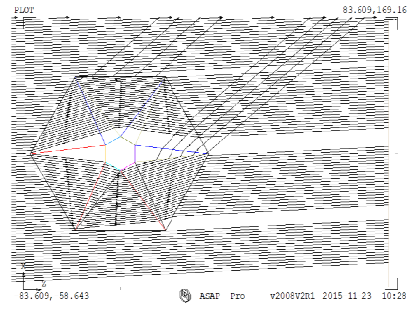

(a) Incident angle deviation: $3^{\circ}$

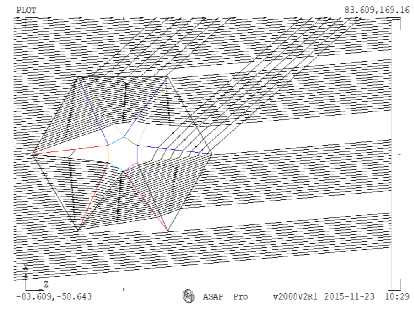

(b) Incident angle deviation: $5^{\circ}$

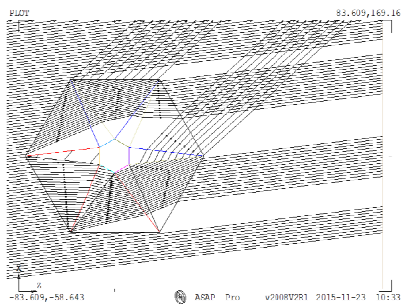

(c) Incident angle deviation: $7^{\circ}$

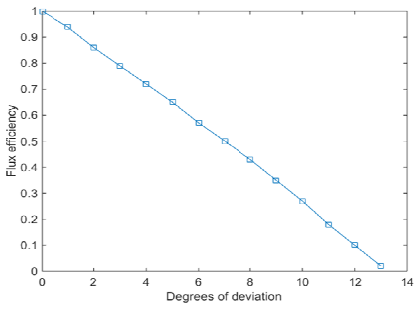

(d) Flux efficiency v.s. degrees of deviation

Figure 6. Rays diagram under different incident angle deviations and the flux efficiency curve 
The above invisible cloak is used under liquid condition, but for terrestrial use, another type of cloak is proposed in reference [5]. The students also constructed a model in ASAP for this type of cloak; the result is shown in Figure 7, with horizontal output rays.

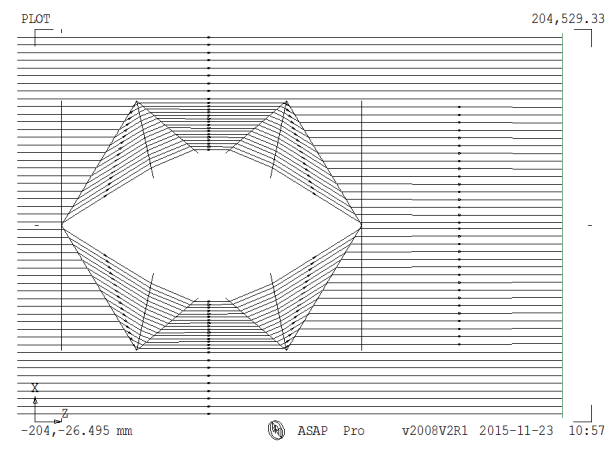

Figure 7. Simulated ray diagram of light passing through the cloak in a terrestrial environment using ASAP

\section{CONCLUSIONS}

Several years of teaching practice of curriculum design and simulation courses shows that, project-based learning meets society needs of training personnel with knowledge, ability and quality. Students have obtained not only skills of using professional software, but also skills of finding and proposing questions in engineering practice, the scientific method of analyzing and solving questions with specialty knowledge, in addition, autonomous learning ability, teamwork spirit and innovation consciousness, still scientific attitude of facing failure and scientific spirit of admitting deficiency in the process of independent design and exploration.

\section{ACKNOWLEDGEMENTS}

We wish to acknowledge financial supports by the Department of Education of Zhejiang Province within "the second batch of '12th Five-Year' Zhejiang provincial experimental teaching demonstration center key construction project" and "the classroom teaching reform and research of Zhejiang provincial higher education project" approved in 2015, and within "the Online and offline mixed teaching mode reform pilot project approved in 2016 by Zhejiang University".

\section{REFERENCES}

[1] Breault Research Organization, " ASAP Primer," 4 August 2014, <http://www.breault.com/knowledge-base/asapprimer?position=0\&list=FCZfZvQVA73xFRfLu6wHjpAGqV9PNsyf7iHKeo-NIlYs > (2017-05-28).

[2] YuanFang Lin, Cheng Zheng, XiaoDong Zheng, XiaoPing, Xu Liu, "Simulation of conoscopic interference in uniaxial crystal using optical software-Advanced System Analysis Program (ASAP)," Opto-Electronic Engineering 39(2), 98-103(2012)

[3] YuanFang Lin, XiaoDong Zheng, Cheng Zheng, XinYang Chen, Xu Liu, "Simulation analysis based on ASAP and experimental verification of the optical image addition and subtraction," Experimental Technology and Management 33(3), 105-111(2016)

[4] YuanFang Lin, XiaoDong Zheng, YiMing Guo, Xu Liu, XiangDong Liu, "Simulations and experiments of holographic grating fabrication optical path adjustment Based on Advanced System Analysis Program (ASAP) ," Research and Exploration in Laboratory 35(5), 91-96(2016)

[5] HongSheng Chen, Bin Zheng, Lian Shen, HuaPing Wang, Xianmin Zhang, Nikolay I. Zheludev, Baile Zhang, "Ray-optics cloaking devices for large objects in incoherent natural light." Nature Communications 4(2652), 1-6(2013) 